\title{
A Two-Scale Damage Model for High Cycle Fatigue Delamination in Laminated Composites
}

\author{
Ahmad Amiri-Rad ${ }^{\mathrm{a}, *}$, Mohammad Mashayekhi ${ }^{\mathrm{a}}$, Frans P. van der Meer ${ }^{\mathrm{b}}$, \\ Homayoun Hadaviniac ${ }^{c}$ \\ ${ }^{a}$ Isfahan University of Technology, Department of Mechanical Engineering, 84156-83111, \\ Isfahan, Iran \\ ${ }^{b}$ Delft University of Technology, Faculty of Civil Engineering and Geosciences, PO Box 5048, \\ 2600 GA Delft, The Netherlands \\ ${ }^{c}$ Kingston University, Department of Mechanical and Automotive Engineering, London SW15 \\ 3DW, United Kingdom
}

\begin{abstract}
In this paper a model for predicting fatigue delamination growth in laminated composites under high cycle fatigue is proposed. The model uses the cohesive zone approach and a two-scale continuum damage mechanics model. The behavior of the interface material is considered quasi-brittle at the macro scale while plastic deformations are allowed at the scale of micro-defects. The validity of the proposed model is investigated through several standard tests using experimental data from literature. Good agreement between the numerical and experimental results is observed. The model is also capable of simulating fatigue under variable amplitude loading. This feature of model is shown through several sample simulations.

Keywords: Cohesive zone model, A. Laminate, B. Fatigue, B. Delamination, C. Damage mechanics
\end{abstract}

\footnotetext{
*Corresponding author

Email address: a.amirirad@me.iut.ac.ir (Ahmad Amiri-Rad)
} 


\section{Introduction}

Many composite structures such as wind turbine blades and aircraft wings experience cyclic loading in their lifetime. Fatigue is one of the most important forms of failure that should be taken into account in designing such structures. Fatigue

5 damage in laminated composites can have several forms such as fiber breakage, matrix cracking, fiber-matrix debonding and delamination. Delamination is separation of layers and can be initiated by low velocity impact, interlaminar stresses near the free edge or manufacturing defects. After delamination is initiated, it can grow under fatigue loading which can lead to a substantial decrease in structural stiffness specially under compressive loads. The ability to predict the delamination growth under fatigue can help in the design process of safe structures.

Several approaches such as methods based on fracture mechanics and cohesive zone methods have been used to simulate the delamination growth. The cohesive zone approach has proven to be a powerful tool for modeling delamination with straightforward numerical implementation. Compared to conventional implementations of fracture mechanics methods, this approach has several advantages such as the ability of modeling initiation and propagation of delamination in a unified way and no need for remeshing after each step of crack growth. In this method a cohesive layer is introduced in potential crack plane. It is assumed that a cohesive process zone exists near the crack tip and in this zone separation of the interface is controlled by a non-linear traction-separation law.

Although use of the cohesive zone method in monotonic loading has been widely explored, less research has been carried out on fatigue loading [1 5] . While most of proposed models are more suitable for cycle by cycle analysis and hence low cy- 
cle fatigue, some phenomenological models more appropriate to high cycle fatigue have been proposed [6, 7]. Several researchers [8, 9] have aimed to find the growth rate of a damage parameter associated with cohesive law using Paris equation. Although these models use Paris equation constants and do not require new material parameters, linking the cohesive zone damage growth to crack growth rate is not straightforward and some assumptions have to be made.

Most methods used for delamination growth like fracture mechanics and cohesive zone approaches are phenomenological methods and do not consider microscopic mechanisms of failure. In these methods well-defined experimentally measured parameters are used to evaluate the structural integrity. In cohesive zone approach energy release rate. The overall effects of microscopic mechanisms are reflected through these parameters and no analysis of these mechanisms is carried out. While incorporating the underlying microscopic behavior in failure analysis provides more insight, it can be computationally challenging. In this paper the common cohesive zone model has been enriched by incorporating microscopic material behavior. As exploring microscopic scale can have many different levels, this model tries to use a simple approach and remain computationally manageable.

\section{Formulation}

In this paper a combination of the cohesive zone model (CZM) and a two-scale damage model (TSDM) for high cycle fatigue is used to model delamination growth. When a laminated composite with an initial delamination is subjected to high cycle fatigue loads, the area surrounding the crack tip will experience damage. 
In the CZM a damage zone is assumed to exist ahead of crack tip which represents this damaged area. During high cycle fatigue this damage will grow gradually and growth and coalescence of micro-defects will result in complete failure of this area and advance of the crack. In the CZM the damaged area ahead of crack tip is called cohesive zone and is represented by elements which have entered into the softening zone in their constitutive equation. Considering this cohesive zone, a proper damage evolution model to simulate the damage growth under cyclic loading is required to have a complete model for predicting delamination growth under fatigue loading. In this paper a two-scale damage model has been used for this purpose. While in low cycle fatigue the damage growth is accompanied with plastic strains, in high cycle fatigue no considerable plastic strains is observed. Although there is no sensible plastic strains at macro-scale, at micro-scale (scale of defects) plastic strains exists [10]. Therefore use of a two-scale damage model which can relate the plastic strain and damage in micro-scale to material behavior in macro-scale seems a reasonable approach [10 12]. In this section the CZM and TSDM approaches will be discussed.

\subsection{Cohesive Zone Model}

The cohesive zone model is one of the most common methods for simulating the delamination phenomenon. This method is based on finite elements and unlike common procedure of fracture mechanics like virtual crack closure technique (VCCT), does not require a remesh after each increment of crack growth. In this method cohesive elements are placed in the expected path of crack growth e.g. between plies in laminated composites.

Cohesive elements are not linear elastic and follow a traction-separation law where 
stress increases linearly from zero to a maximum value (interface strength) and is reduced back to zero at complete material failure. This relation usually includes a damage parameter to progressively reduce the stiffness of the element as interfaces separate. Several traction-separation laws have been suggested [13 17] among which the bi-linear law is most common for composites. The bi-linear tractionseparation law is written as follows:

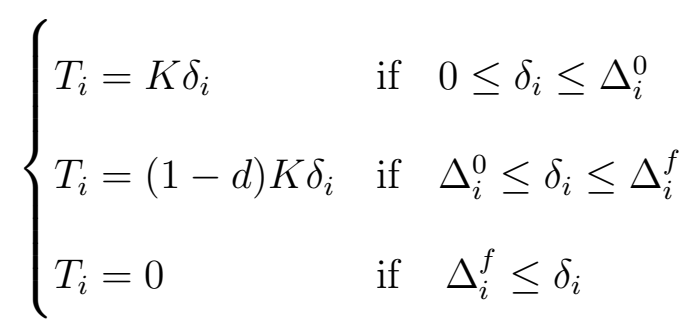

with damage parameter $\mathrm{d}$ as:

$$
d=\frac{\Delta_{i}^{f}\left(\delta_{i}-\Delta_{i}^{0}\right)}{\delta_{i}\left(\Delta_{i}^{f}-\Delta_{i}^{0}\right)}
$$

where $K$ is called penalty stiffness, $\delta_{i}$ and $T_{i}$ are separation and traction in $i$ direction and $\Delta_{i}^{0}$ and $\Delta_{i}^{f}$ are separations at damage initiation and total decohesion in $i$ direction (Figure 1). The area under the traction-separation curve shows the critical energy release rate $G_{c}$.

When the maximum stress in traction-separation law is reached, the damage process and softening starts. When the point is unloaded, the separation will go toward zero and no permanent separation is left. The unloading and reloading paths coincide (Figure 1) and in subsequent reloading the material will behave linearly with the new stiffness value until the new maximum stress is reached. A damage variable which has the value of 0 at the beginning of the softening zone and reaches the value of 1 at the end of this zone can be defined using the ratio be- 
tween rate of dissipated energy and critical energy release rate or between the current and initial maximum stress:

$$
D=\frac{U}{G_{c}}=1-\frac{T}{T^{0}}
$$

where $U$ is the dissipated energy until $\delta$ (Figure 1). This damage parameter has the following relation with damage parameter $d$ used in traction-separation law (Equation 1):

$$
D=1-\frac{\delta}{\Delta^{0}}(1-d)
$$

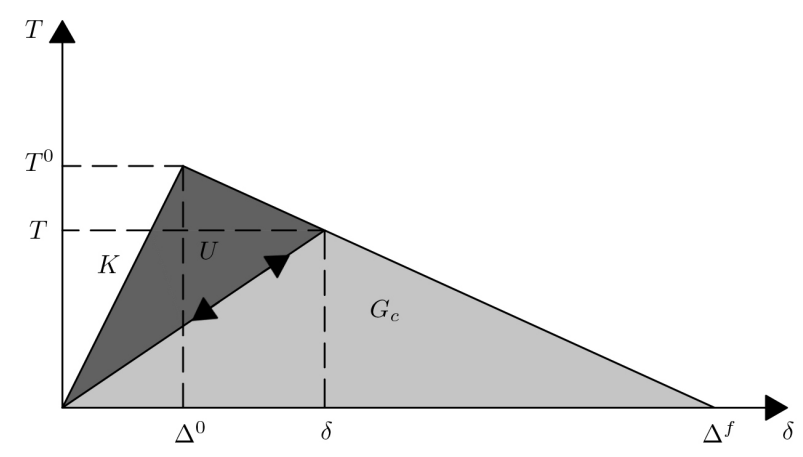

Figure 1: Dissipated energy at separation $\delta$.

From the perspective of continuum damage mechanics, this damage variable is defined as the density of micro-defects in representative volume element (RVE) where with the assumption of isotropic damage it can be taken equal to the ratio of damaged area to total area [10]:

$$
D=\frac{\delta S_{D}}{\delta S}
$$

The stress acting on the remaining area $\left(\delta S-\delta S_{D}\right)$ is called effective stress $\tilde{\sigma}$ and 
has the following relation with stress $\sigma$ :

$$
\tilde{\sigma}=\frac{\sigma}{1-D}
$$

Using this definition and Equation 3, the effective stress in the entire softening zone will be $T^{0}$ :

$$
\tilde{\sigma}=T^{0}=\frac{T}{1-D}
$$

This effective stress will be used as the input to two-scale damage model to calculate the plastic deformation in micro-scale. Distribution of these measures of stress and the cohesive length are shown in Figure 2.

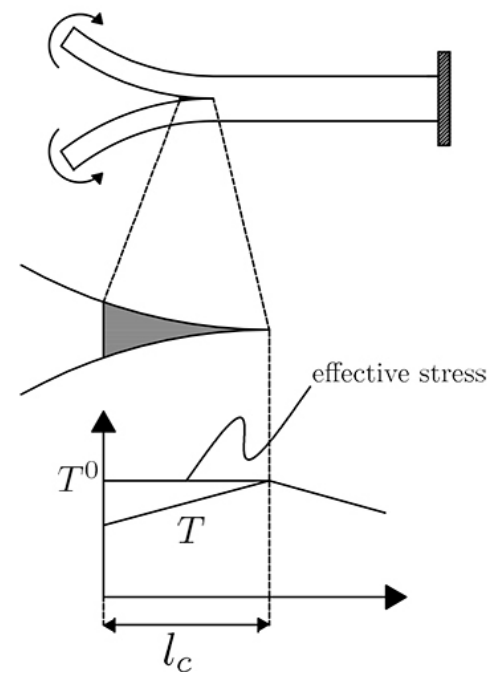

Figure 2: Stress and effective stress distributions in the cohesive zone.

\subsection{Two-Scale Damage Model}

Mechanical properties are presented as homogenized variables over an RVE in continuum mechanics. For example damage parameter as was presented in Equa- 
tion 5 represents all the defects present in the RVE. In this paper two scales are used to model high cycle fatigue [10 12]: 1) the macro-scale which is the classical scale of continuum mechanics and 2)the micro-scale that is the scale of the defects present in the RVE which their effect on the elastic macroscopic behavior is not sensible except for failure. While in ductile failure, low cycle fatigue and creep, considerable plastic deformation occurs at the macro-scale, in quasi-brittle failure and high cycle fatigue no plastic deformation can be observed. However, at the micro-scale, plastic strain is present in such processes [10]. In these cases, plasticity occurs at the micro-scale while macro-scale behaves elastically. This behavior has been taken into account by Lemaitre et al. [10 12 by considering all the micro-defects as a weak inclusion and the macro-scale as a stronger surrounding matrix (Figure 3). The inclusion is called weak since it has lower yield stress than the surrounding matrix and undergoes plastic deformation while the matrix remains elastic. While the yield stress of the surrounding matrix is equal to the yield stress of the material at the macro-scale, the yield stress of the inclusion is taken lower and equal to the fatigue endurance limit of the material. Considering all the defects in a material point as a weak inclusion acts as a homogenization process in the two-scale model. In the current paper this two-scale model has been adopted to use plastic strains in the micro-scale to calculate the fatigue damage growth in an interface material like epoxy which by use of energy release rate and bi-linear traction-separation law is treated as a quasi-brittle material at the macro-scale.

When loads are applied, the inclusion will undergo plastic deformation and tends to experience large strains but the elastic matrix will constraint this deformation. The solution to the problem of deformed inclusion restricted by an elastic 
surrounding matrix was first proposed by Eshelby [18].

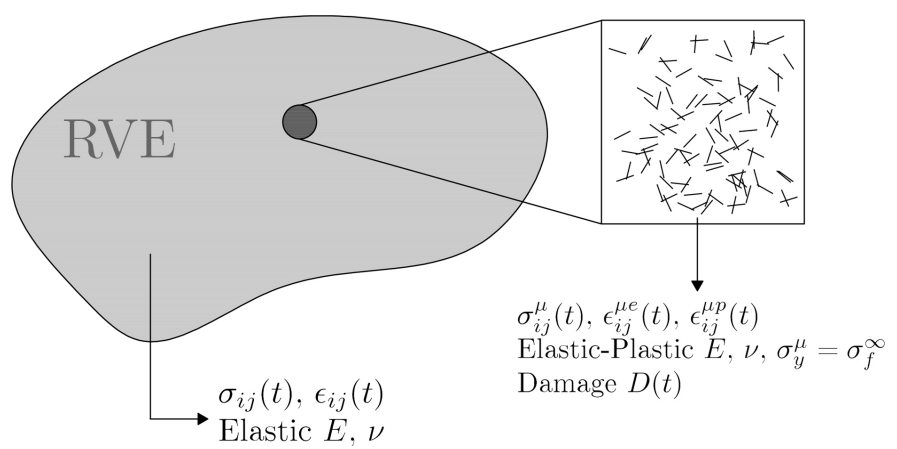

Figure 3: A weak inclusion embedded in an elastic RVE.

In the micro-scale, elastic-plastic behavior with linear kinematic hardening is assumed. In this scale the material is weakened by introducing plastic behavior with a yield stress $\sigma_{y}^{\mu}$ equal to fatigue endurance limit $\sigma_{f}^{\infty}$. The yield criterion is written as follows:

$$
f^{\mu}=\left(\tilde{\boldsymbol{\sigma}}^{\mu}-\boldsymbol{X}^{\mu}\right)_{\mathrm{eq}}-\sigma_{f}^{\infty}
$$

where $\tilde{\boldsymbol{\sigma}}^{\mu}$ and $\boldsymbol{X}^{\mu}$ are effective stress and back stress tensors in the micro-scale and ()$_{e q}$ shows von Mises norm. The superscript $\mu$ is used for quantities at the micro-scale throughout this paper. For simplification only kinematic linear hardening is considered:

$$
\dot{X}_{i j}^{\mu}=\frac{2}{3} C_{y} \dot{\epsilon}_{i j}^{\mu p}\left(1-D_{s}\right)
$$

where $\dot{\epsilon}_{i j}^{\mu p}$ is the rate of the plastic strain tensor, $D_{s}$ is static damage at macroscale described in more detail in section 2.3 and $C_{y}$ is the plastic modulus in microscale and is assumed to be the same as the measured value in the macro-scale. The flow rule has the following form:

$$
\dot{\epsilon}_{i j}^{\mu p}=\frac{3}{2} \frac{\tilde{S}_{i j}^{\mu}-X_{i j}^{\mu}}{\left(\tilde{\boldsymbol{\sigma}}^{\mu}-\boldsymbol{X}^{\mu}\right)_{e q}} \frac{\dot{\lambda}}{1-D_{s}}
$$


where $\tilde{S}_{i j}^{\mu}$ is the deviatoric stress tensor and $\dot{\lambda}$ is the plastic multiplier. The accumulated plastic strain rate is defined as the following norm of the plastic strain tensor rate:

$$
\dot{p}^{\mu}=\sqrt{\frac{2}{3} \dot{\epsilon}_{i j}^{\mu p} \dot{\epsilon}_{i j}^{\mu p}}
$$

The plastic multiplier $\dot{\lambda}=\dot{p}^{\mu}\left(1-D_{s}\right)$ can be obtained using the consistency condition $f^{\mu}=0$ and $\dot{f}^{\mu}=0$.

In the cohesive zone approach the opening of the crack is opposed by cohesive stresses. Here it is assumed that these stresses are applied by very thin resin layer between two interfaces or laminas. The reaction of these cohesive stresses will be applied by the interface to the resin with the same magnitude and in the opposite direction. Having these forces applied on the resin, an approximation for stress state in the resin has been found. The normal stress component in direction normal to mode I direction is ignored in the cohesive models because of its small contribution to energy release rate and damage. Other stress components associated with plane strain loading conditions may appear in the macro-scale. Plane strain condition in the macro-scale would not necessarily lead to the same condition in the micro-scale and nonzero out-of-plane strain component may also appear in this scale. Because of the small thickness of the resin, it is assumed that the stresses do not vary along the thickness. The Eshelby-Krnoer localization law is used for the scale transition. Total and plastic strains at the macro-scale are related to strains at the micro-sclae through the following equation:

$$
\epsilon_{i j}^{\mu}=\epsilon_{i j}+\beta\left(\epsilon_{i j}^{\mu p}-\epsilon_{i j}^{p}\right)
$$

the term $\epsilon_{i j}^{p}$ is set to zero since there exists no plastic strain at the macro-scale 
and the total strain at the macro scale $\epsilon_{i j}$ is calculated from stresses at this scale using elastic compliance tensor. For a spherical inclusion $\beta$ is given by Eshelby's analysis as:

$$
\beta=\frac{2}{15} \frac{4-5 \nu}{1-\nu}
$$

where $\nu$ is Poisson's ratio.

\subsection{Fatigue Damage Evolution}

Damage created by the application of cyclic loading can be divided into two parts:

initial damage caused by amplitude of applied load and the damage accumulated by repetition of this amplitude. These two damage components are respectively shown by $D_{s}$ and $D_{f}$ in the following equation:

$$
D=D_{s}+D_{f}
$$

The $D_{s}$ component can be calculated from Equation 3 after finding the status of the cohesive zone through quasi-static analysis of the specimen subjected to a load equal to the amplitude. The $D_{f}$ component will be found through damage evolution equation that will be introduced in this section.

While cycle by cycle analysis is appropriate for low cycle fatigue, it is computationally prohibitive for high cycle fatigue which involves a number of cycles of the order $10^{5}$ or more. A strategy that is used as a remedy is the cycle jump method which compromises precision in exchange for a reduction in computation time. In this approximation it is assumed that the damage growth or crack growth rate remains constant over a number of cycles. If the damage growth rate at cycle $N$ is 
$\frac{d D}{d N}$, damage after $\Delta N$ cycles after this point will be:

$$
D_{N+\Delta N}=\left.\frac{d D}{d N}\right|_{N} \Delta N+D_{N}
$$

Since current work is oriented toward high cycle fatigue analysis, use of such a strategy is justifiable. Having this tradeoff between precision and computation cost in mind, instead of using a damage growth model for each point in the cohesive zone, a damage parameter for entire cohesive zone is proposed, since the former involves more complexity and will lose its superiority in precision by use of the cycle jump method. In this method it will be assumed that the whole cohesive zone will fail at the same rate (because they all experience same effective stress according to Equation 7) and since cohesive zone is small in high cycle fatigue, this will not require a big $\Delta N$. A single value for $D_{s}$ will be used for the whole cohesive zone which is the average of $D_{s}$ values at different integration points in this zone. After the whole cohesive zone fails, the crack advances to the extent of this zone and the analysis is repeated for the newly formed cohesive zone . Integrating the TSDM set of equations using $D_{s}$ damage value, the accumulated plastic strain $\Delta p^{\mu}$ (Equation 11) over once loading cycle can be calculated. Using this value we propose the following equation for damage evolution of the cohesive zone:

$$
\frac{d D_{f}}{d N}=\alpha\left(\Delta p^{\mu} l_{c}\right)^{\beta}
$$

where $\Delta p^{\mu}$ is the accumulated plastic strain in one loading cycle and $l_{c}$ is the length of the cohesive zone. Material parameters $\alpha$ and $\beta$ are found through curve fitting. Dependence of the damage growth on the length of the cohesive zone be- 
side permanent deformation in micro-scale can be justified by non-local damage theory. While this theory has been used to avoid local softening and mesh dependency in implementation of continuum damage mechanics, it is more than just a numerical remedy and has a sound physical interpretation. While according to the principal of local action which is vastly used in continuum mechanics, damage at each point only depends on the strain state of that point, the non-smooth distribution of strain in the micro-structure makes the use of non-local damage models more justifiable. In these models, the effects of strain in neighboring points are also included in the damage evolution. Likewise in the model proposed here, there is a dependency between the damage growth rate and the extent of the plastic zone around each point.

\section{Results and Discussion}

The cohesive element formulation has been implemented through a user element subroutine (UEL) in ABAQUS ${ }^{\circledR}[19]$. The model containing standard plane strain elements and these cohesive elements, is solved prior to applying the fatigue load. From this analysis the damage induced by the cyclic load amplitude i.e. $D_{S}$ in Equation 14 and the length of cohesive zone associated with this load is found. The plastic strain in each cycle is calculated by integrating the TSDM set of equations using Elastic predictor-plastic corrector scheme. This value and the cohesive zone length will be used to calculate the fatigue damage accumulation (Equation 16.).

To investigate the performance of the present model under mode I and II, delamination growth in a double cantilever beam (DCB) and a 4-point end notch flexure (4ENF) specimens are simulated and results are compared with experimental re- 
sults reported by Asp et. al [20]. The experiments were performed on HTA/6376C carbon/epoxy with $\left[0_{12} / /\left( \pm 5 / 0_{4}\right)_{s}\right]$ layup where // refers to plane where initial crack is introduced. The specimen has width $b=20 \mathrm{~mm}$, length $l=150 \mathrm{~mm}$, thickness $2 h=3.1 \mathrm{~mm}$ and crack length $a_{0}=35 \mathrm{~mm}$. The specimen geometry is shown in Figure 4. Material properties for this specimen are taken from [20, 21] (see Table 1). Values of $C_{y}=\frac{E_{3}}{100}$ and $\sigma_{f}^{\infty}=\frac{\sigma_{y}}{3}$ are chosen for the plastic modulus and the fatigue endurance limit of the resin material.

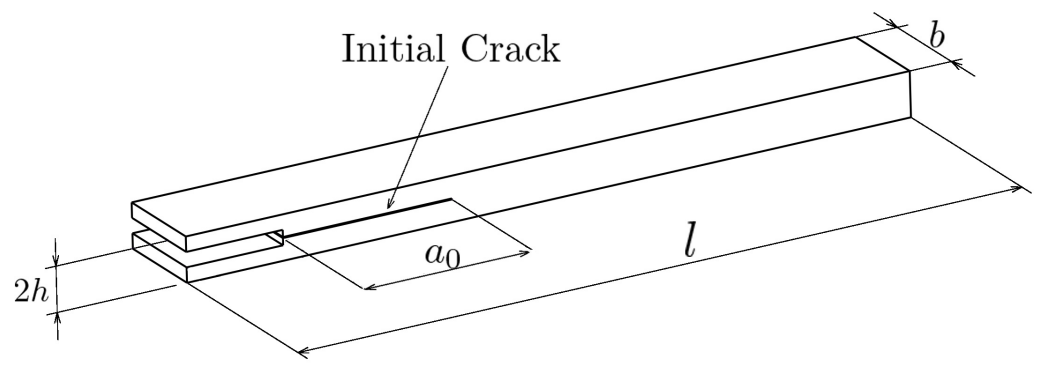

Figure 4: Double cantilever beam specimen dimensions.

Table 1: Material Properties for HTA/6376C carbon/epoxy [20, 21].

\begin{tabular}{ll|ll}
\hline Property & Value & Property & Value \\
\hline$E_{11}(\mathrm{GPa})$ & 120 & $G_{I c}\left(\mathrm{~kJ} / \mathrm{m}^{2}\right)$ & 0.260 \\
$E_{22}=E_{33}(\mathrm{GPa})$ & 10.5 & $G_{I I c}\left(\mathrm{~kJ} / \mathrm{m}^{2}\right)$ & 1.002 \\
$G_{12}=G_{13}(\mathrm{GPa})$ & 5.25 & $K\left(\mathrm{~N} / \mathrm{mm}^{3}\right)$ & $10^{6}$ \\
$G_{23}(\mathrm{GPa})$ & 3.48 & $T_{I}^{0}(\mathrm{MPa})$ & 30 \\
$\nu_{12}=\nu_{13}$ & 0.3 & $T_{I I}^{0}=T_{I I I}^{0}(\mathrm{MPa})$ & 30 \\
$\nu_{23}$ & 0.51 & & \\
\hline
\end{tabular}

The loading pattern for mode I (DCB) and mode II (4ENF) specimens are shown in Figure 5. Since the intention is to compare the results with previous works which have used Paris equation, the strain energy release rate has been computed for each loading. The Paris equation can be written in terms of energy release rate 
as following:

$$
\frac{d a}{d N}=C\left(\frac{\Delta G}{G_{c}}\right)^{m}
$$

where $a$ is crack length, $N$ is number of load cycles, $\Delta G$ is the cyclic change in energy release rate, $G_{c}$ is critical energy release rate and $C$ and $m$ are material parameters. For the material under study, $C$ and $m$ for mode I are obtained respectively $0.0031 \mathrm{~mm} /$ cycle, and 5.4 from results provided in [20].

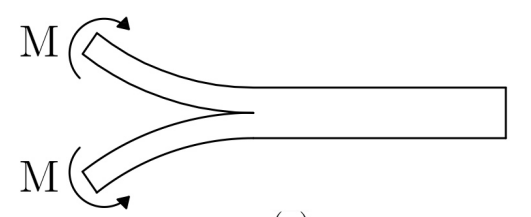

(a)

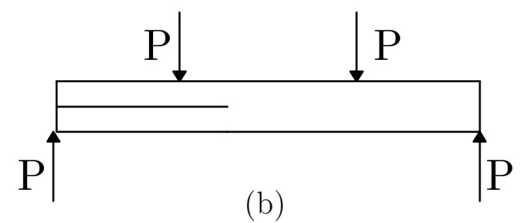

Figure 5: Loading patterns for (a) DCB and (b) 4ENF specimens

The crack growth rate $(d a / d N)$ vs. $G_{I} / G_{I c}$ is plotted in Figure 6 -a for results obtained from the present model and from the experiments [20]. The Paris curve fitted to experimental data has also been plotted. Numerical and experimental results are in good agreement for fitting parameters of $\alpha=3.48 \times 10^{15} \frac{1}{\text { cycle }(m m)^{\beta}}$ and $\beta=8.51$.

Paris equation (Equation 17) constants for mode II for experimental results presented in [20], are $C=0.15 \mathrm{~mm} /$ cycle and $m=4.5$.Using these constants, Paris equation is plotted in Figure 6-b. Choosing fitting parameters value of $\alpha=$ $2.4 \times 10^{6} \frac{1}{\text { cycle }\left(\mathrm{mm}^{2}\right)^{\beta}}$ and $\beta=5.86$ a very good agreement between numerical and experimental results is observed. 


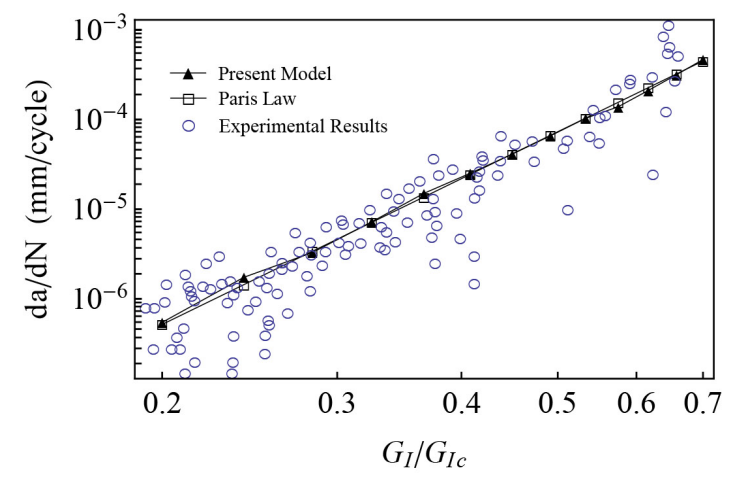

(a)

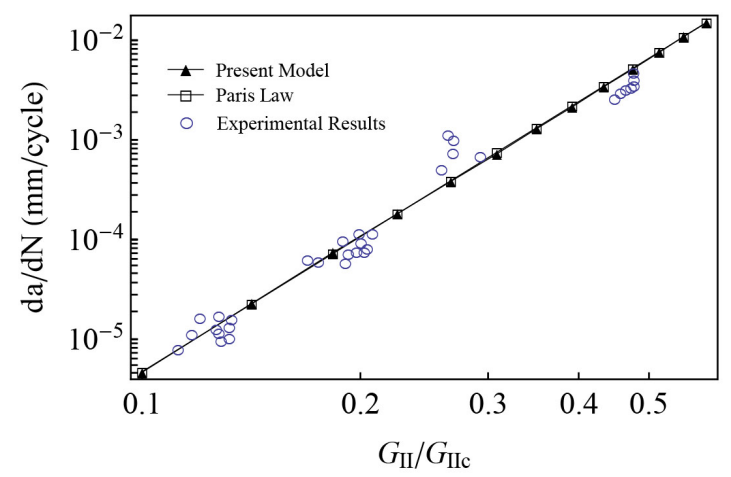

(b)

Figure 6: Crack growth rate vs. energy release rate ratio for (a) mode I and (b) mode II from numerical results of present model and experimental results of 20 .

The fatigue crack advance is due to accumulation of damage in the cohesive zone at the tip of the crack. This damage accumulation consists of nucleation, growth and coalescence of micro-defects such as micro-cracks, micro-crazes and microvoids. These micro-defects are small compared to the scale of representative volume element (RVE) used in definition of damage variable in damage mechanics, therefore they are treated as a smaller, separated scale. The growth of these micro-defects is accompanied by plastic deformation and in the current work this plastic deformation is used to calculate rate of damage evolution. The stress-strain relation for a complete cycle at the micro and macro scale with load ratio $R=0$ is shown in Figure 7 a. The stress at the macro-scale follows a linear path $\mathrm{ABC}^{\prime} \mathrm{A}$ from beginning to the end of a cycle. Simultaneously the micro-scale stress experiences nonlinear behavior with kinematic strain hardening along ABCDA path. The change in accumulated plastic strain at micro-scale during a cycle is shown in Figure $7 \mathrm{~b}$.

Many composite structures such as wind turbine blades and aircraft wings experience variable amplitude loading during their lifetime. The cohesive zone modeling 


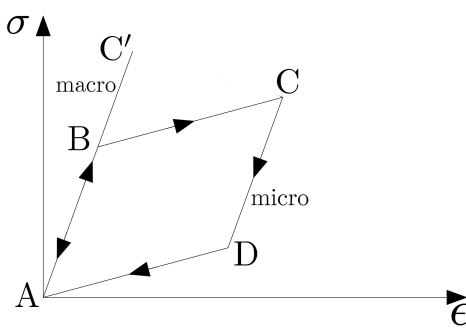

(a)

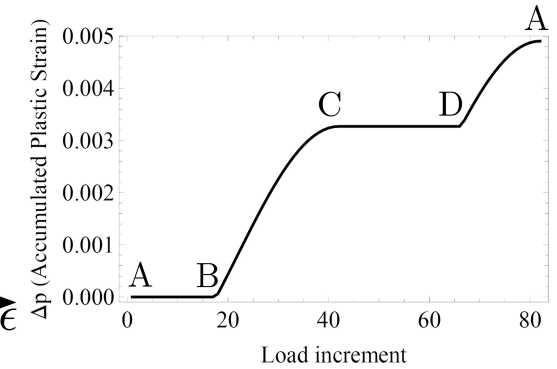

(b)

Figure 7: (a) stress-Strain variation in micro and macro scales (b) accumulated plastic strain in micro-scale.

is a good tool to handle variable amplitude loading histories since it doesn't re-

quire remeshing after each step of crack growth. To test the performance of the model under variable amplitude loading, three different loads levels $P_{1}, P_{2}$ and $P_{3}$ have been chosen. Each of these loads have been applied for respectively $N_{1}, N_{2}$ and $N_{3}$ cycles and the crack growth has been calculated. For values of load and cycles shown in Table 2 the obtained crack growth length is $1.43 \mathrm{~mm}$ and has difference of $5.68 \%$ with value calculated using Paris equation. From the 13 loads shown in Figure 6 with markers, all possible combinations of 3 different values are chosen and for simplicity the loads are applied in order of biggest to smallest. All the 286 possible choices of 3 loads, and their error are shown in Figure 8. It can be seen from this figure the maximum error is about $22 \%$.

Table 2: Load values and number of cycles for variable amplitude loading.

\begin{tabular}{llllll}
\hline$N_{1}$ & $P_{1}$ & $N_{2}$ & $P_{2}$ & $N_{3}$ & $P_{3}$ \\
\hline 3103 & $776 N$ & 1551 & $890.26 N$ & 663 & $997.03 N$
\end{tabular}

A loading history containing different loading amplitudes randomly chosen from 13 loads shown by markers in Figure 6 is produced. This loading history is shown in Figure 9-a. The damage growth for this loading history has been obtained us- 


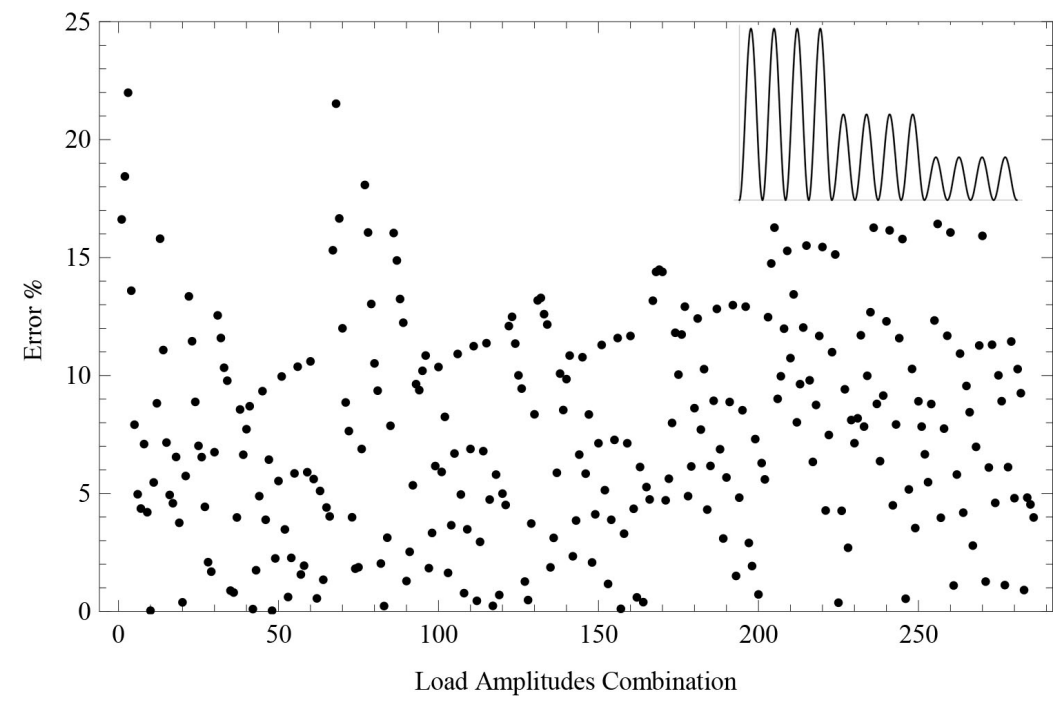

Figure 8: Errors in calculated crack growth for different combinations of three load values.

ing the present model and is illustrated in Figure 9 $\mathrm{b}$.

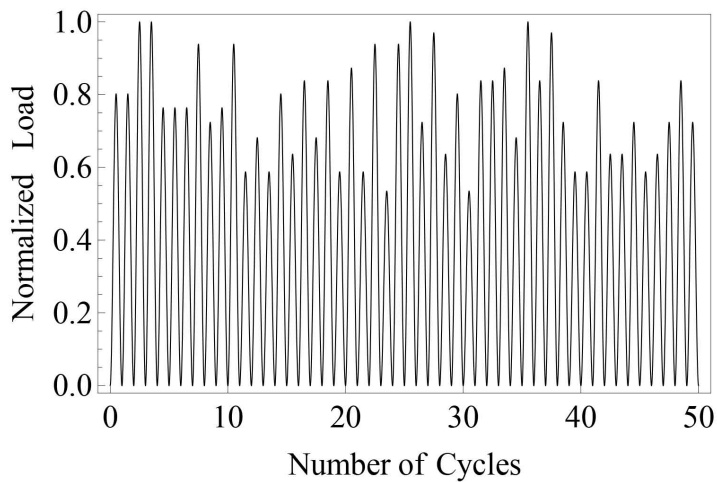

(a)

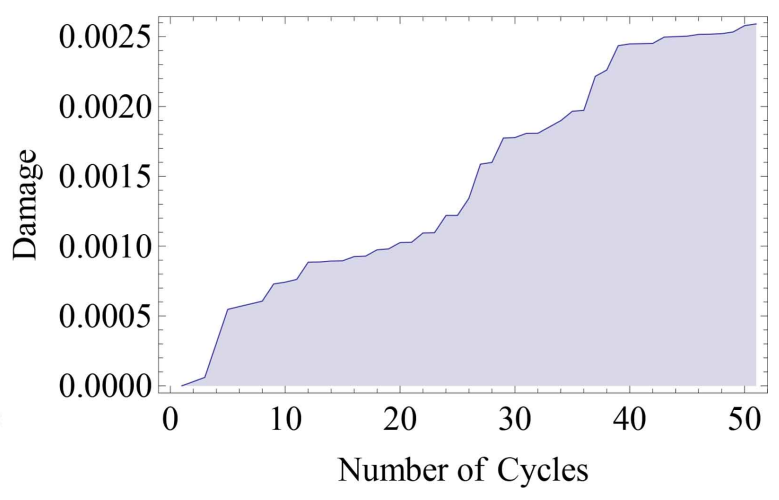

(b)

Figure 9: (a) A loading history with random varying amplitudes (b) Damage growth vs. number of load cycles .

In actual applications usually there is no pure mode I or mode II loading and a mixture of these loading modes may be present. A mixed-mode loading scenario is specified by its mode ratio which is defined as the following ratio at the edge of 
the cohesive zone which is the most highly damaged point of this zone:

$$
B=\frac{\delta_{I I}}{\lambda}
$$

where $\delta_{I I}$ is the separation in mode II and $\lambda=\sqrt{\delta_{I}^{2}+\delta_{I I}^{2}}$ is the mixed-mode equivalent separation. In Figure 10 parameters $\alpha$ and $\beta$ are plotted for different mode ratios, where $B=0$ and $B=1$ correspond to pure mode I and mode II respectively. The parameters for mixed-mode ratio of 0.42 are calculated by simulation of fatigue crack growth in a mixed-mode specimen and fitting the results to the experimental data from [20]. The non-monotonic change in $\beta$ with respect to mode-ratio is also recognized by Blanco et al. 22] for Paris equation constants. In Figure 10 a second order polynomial is fitted to the data. Values for other mode ratios can be found using this interpolation. Having access to experimental data for more mixed-mode ratios can lead to more accurate interpolation.
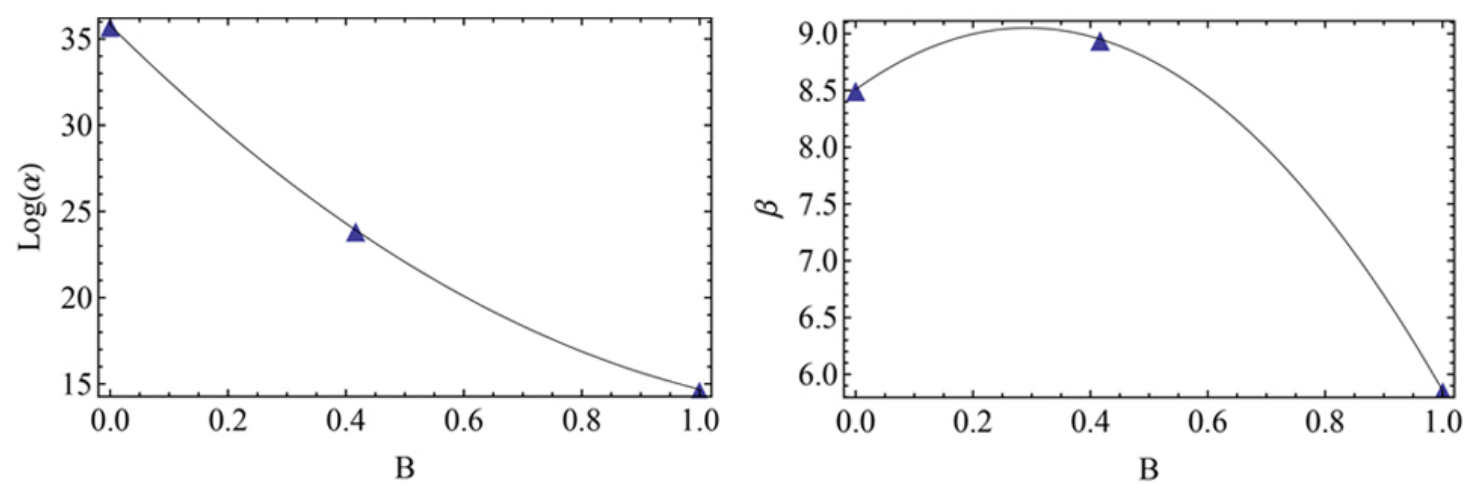

Figure 10: Variation of model parameters with mode ratio.

\section{Conclusion}

A new approach for fatigue delamination growth prediction in laminated composites was proposed. The cohesive zone model along with a two-scale damage model 
was used to link the material behavior at macro and micro scales. The damage in the softened zone surrounding the crack tip was divided in two parts: a part responsible for the damage induced by the amplitude of cyclic loading and a part representing the damage caused by the repetition of this amplitude. The growth of the later component per cycle was related to the accumulated plastic strain in micro-scale during the cycle and the length of the cohesive zone. The proposed model tries to avoid high computational cost of high cycle fatigue simulation by using a strategy based on cycle jump method. The model was successfully used to simulate the experiments presented in literature for pure mode I and mode II delamination under constant amplitude cyclic loading. The model parameters for mode I, mode II and mixed mode loading were obtained; dependence of the damage model parameters on loading mode can be eliminated by further understanding of the fatigue damage mechanism. While the accuracy of some of the model assumptions can be improved with more experimental data available, the proposed approach can be considered as a framework for multi-scale fatigue damage analysis. It was shown that the model is able to handle the variable amplitude loading with a relatively good accuracy. A numerical example was used to show the ability of the model with variable amplitude loading. However further experimental data is needed to validate this aspect of the model.

\section{References}

[1] J. Foulk, D. Allen, K. Helms, A model for predicting the damage and environmental degradation dependent life of scs-6/timetal@ 21s [0] 4 metal matrix composite, Mechanics of materials 29 (1) (1998) 53-68.

[2] A. De-Andrés, J. Pérez, M. Ortiz, Elastoplastic finite element analysis of three-dimensional 
fatigue crack growth in aluminum shafts subjected to axial loading, International journal of solids and structures 36 (15) (1999) 2231-2258.

[3] B. Yang, S. Mall, K. Ravi-Chandar, A cohesive zone model for fatigue crack growth in quasibrittle materials, International journal of solids and structures 38 (22) (2001) 3927-3944.

[4] O. Nguyen, E. Repetto, M. Ortiz, R. Radovitzky, A cohesive model of fatigue crack growth, International Journal of Fracture 110 (4) (2001) 351-369.

[5] K. Roe, T. Siegmund, An irreversible cohesive zone model for interface fatigue crack growth simulation, Engineering fracture mechanics 70 (2) (2003) 209-232.

[6] P. Robinson, U. Galvanetto, D. Tumino, G. Bellucci, D. Violeau, Numerical simulation of fatigue-driven delamination using interface elements, International journal for numerical methods in engineering 63 (13) (2005) 1824-1848.

[7] J. Munoz, U. Galvanetto, P. Robinson, On the numerical simulation of fatigue driven delamination with interface elements, International Journal of Fatigue 28 (10) (2006) 11361146.

[8] A. Turon, J. Costa, P. Camanho, C. Dávila, Simulation of delamination in composites under high-cycle fatigue, Composites Part A: applied science and manufacturing 38 (11) (2007) 2270-2282.

[9] P. W. Harper, S. R. Hallett, A fatigue degradation law for cohesive interface elementsdevelopment and application to composite materials, International Journal of Fatigue 32 (11) (2010) 1774-1787.

[10] J. Lemaitre, R. Desmorat, Engineering damage mechanics: ductile, creep, fatigue and brittle failures, Springer, 2005.

[11] J. Lemaitre, I. Doghri, Damage 90: a post processor for crack initiation, Computer methods in applied mechanics and engineering 115 (3) (1994) 197-232. 
[12] J. Lemaitre, J. Sermage, R. Desmorat, A two scale damage concept applied to fatigue, International Journal of Fracture 97 (1-4) (1999) 67-81.

[13] A. Needleman, A continuum model for void nucleation by inclusion debonding, Journal of Applied Mechanics 54 (3) (1987) 525-531.

[14] X.-P. Xu, A. Needleman, Numerical simulations of fast crack growth in brittle solids, Journal of the Mechanics and Physics of Solids 42 (9) (1994) 1397-1434.

[15] A. Needleman, An analysis of intersonic crack growth under shear loading, Journal of Applied Mechanics 66 (4) (1999) 847-857.

[16] Y. Mi, M. Crisfield, G. Davies, H. Hellweg, Progressive delamination using interface elements, Journal of composite materials 32 (14) (1998) 1246-1272.

[17] J. Williams, H. Hadavinia, Analytical solutions for cohesive zone models, Journal of the Mechanics and Physics of Solids 50 (4) (2002) 809-825.

[18] J. D. Eshelby, The determination of the elastic field of an ellipsoidal inclusion, and related problems, Proceedings of the Royal Society of London. Series A. Mathematical and Physical Sciences 241 (1226) (1957) 376-396.

[19] H. Hibbitt, B. Karlsson, P. Sorensen, Abaqus analysis users manual version 6.10, Dassault Systèmes Simulia Corp.: Providence, RI, USA.

[20] L. E. Asp, A. Sjgren, E. S. Greenhalgh, Delamination growth and thresholds in a carbon/epoxy composite under fatigue loading, Journal of Composites Technology \& Research $23(2)(2001) 55-68$.

[21] M. Juntti, L. E. Asp, A. Olsson, Assessment of evaluation methods for the mixed-mode bending test, Journal of Composites Technology \& Research 21 (1) (1999) 37-48.

[22] N. Blanco, E. K. Gamstedt, L. Asp, J. Costa, Mixed-mode delamination growth in carbonfibre composite laminates under cyclic loading, International journal of solids and structures $41(15)(2004)$ 4219-4235. 